\title{
Complex modified generalized projective synchronization of fractional-order complex chaos and real chaos
}

\author{
Jian Liư ${ }^{*}$, Shutang Liu² and Wei Li ${ }^{3}$
}

\section{"Correspondence:}

liujian1990@163.com

'School of Mathematical Sciences,

University of Ji'nan, Ji'nan,

Shandong 250022, P.R. China

Full list of author information is

available at the end of the article

\section{然 Springer}

\begin{abstract}
This paper introduces a type of modified generalized projective synchronization with complex transformation matrix (CMGPS) for fractional-order complex chaos and real chaos with the same dimension and different structures. The transformation matrix in this type of chaos synchronization is a non-diagonal square matrix, and its elements are complex numbers. Based on the stability theory of fractional-order systems, necessary and sufficient criteria are established to guarantee CMGPS for the fractional-order complex chaos and fractional-order real chaos, and for two fractional-order complex chaotic systems, respectively. Numerical examples are provided to illustrate the feasibility and effectiveness of our theoretical results.
\end{abstract}

PACS Codes: $05.45 . G g ; 05.45 . X t ; 05.45 . P q$

Keywords: fractional-order chaos; chaos with complex variable; modified generalized projective synchronization

\section{Introduction}

In the last thirty years, with the development of interdisciplinary applications, it was found that many systems in interdisciplinary fields can be elegantly described with the help of fractional derivatives, for instance, viscoelastic systems [1], dielectric polarization [2], quantitative finance [3], quantum evolution of complex systems [4], and so forth. Due to the above wide scope of applications, many researchers devoted much effort to chaotic behaviors, chaotic control, and synchronization of fractional-order dynamical systems in a real space. For example, Hartley et al. introduced the fractional-order continuous ChuaHartley's system [5], Arena et al. considered the fractional-order cellular neural network [6], Gao and Yu presented the fractional continuous Duffing's systems [7], Wu et al. addressed discrete chaos and synchronization of the fractional logistic, sine and standard maps recently [8-10]. The projective synchronization (PS) [11] has been especially extensively studied because it can be used to obtain faster communication with its proportional feature, and the unpredictability of the scaling factor can additionally enhance the security of communication. In [12], Wu and Lu presented a modified projective synchronization (MPS) method for fractional-order Chen hyperchaotic systems, which associates with the projective synchronization and the generalized one, where the drive and response systems could be synchronized up to scaling factors $\delta_{i}$. Liu et al. [13] introduced modified general-

(c) 2015 Liu et al. This article is distributed under the terms of the Creative Commons Attribution 4.0 International License (http://creativecommons.org/licenses/by/4.0/), which permits unrestricted use, distribution, and reproduction in any medium, provided you give appropriate credit to the original author(s) and the source, provide a link to the Creative Commons license, and indicate if changes were made. 
ized projective synchronization (MGPS) of fractional-order chaotic systems with different structure, where the drive and response systems could be asymptotically synchronized up to a desired non-diagonal transformation matrix.

In 2013, several research results were proposed about the dynamic properties and synchronization of fractional-order chaotic systems in a complex space. The fractional-order complex Lorenz system was proposed and dynamics of the system was investigated in detail [14]. Luo and Wang introduced the fractional-order complex Chen system and its application to digital secure communication, the complex variables (doubling the number of variables) increase the content of transmitting information signals and enhance their security further [15].

However, all the scaling factors in the above synchronization are real numbers. That is to say, the drive and response systems evolve in the same or inverse direction simultaneously. In fact, for complex dynamical systems, the scaling factors can be complex [16-19], the drive and response systems may evolve in different directions with a constant intersection angle, for example, $\zeta=\rho e^{j \gamma} \eta$, where $\rho e^{j \gamma}=\rho(\cos \gamma+j \sin \gamma), \zeta$ and $\eta$ denote the complex state variables of drive and response systems, respectively, $\rho>0$ denotes the zoom rate, $\gamma \in[0,2 \pi)$ denotes the rotate angle. Moreover, as the complex scaling factors are arbitrary and more unpredictable than real scaling factors and the operations of complex numbers are complicated, the possibility that an interceptor extracts the information from the transmitted signal is greatly less than real scaling factors, which will also increase security and variety of communications. However, complex synchronization of fractional-order complex chaos and fractional-order real chaos is less. Only in [20], Liu introduced modified hybrid projective synchronization with complex transformation matrix (CMHPS) for different dimensional fractional-order complex chaos and fractional-order real hyper-chaos. Naturally, a question may be put forth: Does there exist another kind of complex synchronization, where the same dimensional fractional-order complex chaos and real chaos could be synchronized up to a non-diagonal complex transformation matrix $\Lambda=\Lambda^{r}+j \Lambda^{i}$ ? In the practical applications, there does exist this type of synchronization, called modified generalized projective synchronization with complex transformation matrix (CMGPS). By means of the complex state transformation matrix, every state variable in the response system will be involved in multiple state variables of the drive system, which will increase the complexity of the synchronization and further increase the diversity and the security of communications. Therefore, it is interesting and significant to study CMGPS of two fractional-order complex chaotic systems and that of fractional-order complex chaos and real chaos. However, to the best of our knowledge, this type of CMGPS for fractional-order chaotic systems has rarely been reported.

Motivated by the above discussion, CMGPS is addressed for fractional-order complex chaos and real chaos with the same dimension and different structures based on the stability theory of fractional-order systems. In addition, CMGPS will contain MPS with real constant scaling matrix and MGPS with real transformation matrix and extend previous works.

The rest of this paper is organized as follows. In Section 2, a brief review of the fractional derivative and numerical algorithm and the stability theory of the fractional-order system is given. General methods of CMGPS for the fractional-order real chaotic drive system and the complex chaotic response system, for the fractional-order complex chaotic drive system and the real chaotic response system, and for two fractional-order complex chaotic 
systems are presented in Section 3, Section 4 and Section 5, respectively. Three numerical examples are provided in Section 6. Finally, some conclusions are drawn in Section 7.

Notations $\mathbb{R}^{n}$ stands for $n$-dimensional real vector space, $\mathbb{C}^{n}$ stands for $n$-dimensional complex vector space. If $z \in \mathbb{C}^{n}$ is a complex vector, then $z=z^{r}+j z^{i}, j=\sqrt{-1}$ is the imaginary unit, superscripts $r$ and $i$ stand for the real and imaginary parts of $z$, respectively, $z^{\mathrm{T}}$ are the transpose of $z$, respectively, and $\|z\|$ implies the 2-norm of $z$. If $z$ is a complex scalar, $\bar{z}$ is the conjugate of $z$.

Assume $\alpha>0$, then $\lceil\alpha\rceil$ is just the value $\alpha$ rounded up to the nearest integer, $J^{\alpha}$ denotes Riemann-Liouville type fractional integral of order $\alpha, D^{\alpha}$ denotes Riemann-Liouville type fractional derivative of order $\alpha, D_{*}^{\alpha}$ denotes Caputo type fractional derivative of order $\alpha$, $\Gamma(\cdot)$ denotes the gamma function $\Gamma(x)=\int_{0}^{\infty} t^{x-1} e^{-t} d t, x>0$.

\section{Preliminaries}

\subsection{The definition of fractional derivative}

There are many definitions of fractional derivative [21]. The definition of the RiemannLiouville derivative is given as

$$
D^{\alpha} f(t)=\frac{d^{m}}{d t^{m}} J^{m-\alpha} f(t)
$$

where $\alpha>0, m:=\lceil\alpha\rceil, J^{\beta}$ is the $\beta$-order Riemann-Liouville integral operator as described by

$$
J^{\beta} f(t)=\frac{1}{\Gamma(\beta)} \int_{0}^{t} \frac{f(\tau)}{(t-\tau)^{1-\beta}} d \tau,
$$

where $0<\beta \leq 1$.

The Caputo fractional derivative is defined as

$$
D_{*}^{\alpha} f(t)=\frac{d^{m}}{d t^{m}} J^{m-\alpha} f(t)= \begin{cases}\frac{1}{\Gamma(m-q)} \int_{0}^{t} \frac{f^{(m)}(\tau)}{(t-\tau)^{(q-m+1)}} d \tau, & m-1<q<m, \\ \frac{d^{m}}{d t^{m}} f(t), & q=m,\end{cases}
$$

where $\alpha>0, m:=\lceil\alpha\rceil$.

Generally speaking, there are two numerical methods suitable for chaos synchronization of fractional differential systems. One is the frequency-domain method [22], another is the time-domain method. Here, the Caputo version and an improved predictor-corrector algorithm, i.e., the Adams-Bashforth-Moulton predictor-correctors scheme are chosen for fractional differential equations, where the numerical approximation is a time-domain approach that is more accurate, and the computational cost is greatly reduced [23, 24].

\subsection{Numerical algorithms}

The fractional predictor-corrector algorithm [23] is based on the analytical property of the following fractional differential equation:

$$
\begin{cases}D_{*}^{\alpha} x(t)=f(t, x(t)), & 0 \leq t \leq T, \\ x^{(k)}(0)=x_{0}^{(k)}, & k=0,1, \ldots, m-1(m=\lceil\alpha\rceil),\end{cases}
$$


which is equivalent to the Volterra integral equation

$$
x(t)=\sum_{k=0}^{m-1} x_{0}^{(k)} \frac{t^{k}}{k !}+\frac{1}{\Gamma(\alpha)} \int_{0}^{t} \frac{f(\tau, x(\tau))}{(t-\tau)^{1-\alpha}} d \tau .
$$

Now, set $h=T / N, t_{n}=n h\left(n=0,1,2, \ldots, N \in Z^{+}\right)$. Equation (5) can be written as

$$
\begin{aligned}
x_{h}\left(t_{n+1}\right)= & \sum_{k=0}^{m-1} x_{0}^{(k)} \frac{t_{n+1}^{k}}{k !}+\frac{h^{\alpha}}{\Gamma(\alpha+2)} f\left(t_{n+1}, x_{h}^{\theta}\left(t_{n+1}\right)\right) \\
& +\frac{h^{\alpha}}{\Gamma(\alpha+2)} \sum a_{j, n+1} f\left(t_{j}, x_{h}\left(t_{j}\right)\right),
\end{aligned}
$$

where the predicted value $x_{h}^{\theta}\left(t_{n+1}\right)$ is determined by

$$
\begin{aligned}
x_{h}^{\theta}\left(t_{n+1}\right)= & \sum_{k=0}^{m-1} x_{0}^{(k)} \frac{t_{n+1}^{k}}{k !}+\frac{h^{\alpha}}{\Gamma(\alpha+2)} f\left(t_{n+1}, x_{h}^{\theta}\left(t_{n+1}\right)\right) \\
& +\frac{h^{\alpha}}{\Gamma(\alpha+2)} \sum b_{j, n+1} f\left(t_{j}, x_{h}\left(t_{j}\right)\right),
\end{aligned}
$$

and

$$
\begin{aligned}
& a_{j, n+1}= \begin{cases}n^{\alpha+1}-(n-\alpha)(n+1)^{\alpha+1}, \\
(n-j+2)^{\alpha+1}+n-j^{\alpha+2}-2(n-j+1)^{\alpha+1}, & 1 \leq j \leq n,\end{cases} \\
& b_{j, n+1}=\frac{h^{\alpha}}{\alpha}\left((n-j+1)^{\alpha}-(n-j)^{\alpha}\right) .
\end{aligned}
$$

The estimation error in this method is

$$
\varepsilon=\max \left(\left|x\left(t_{j}\right)-x_{h}\left(t_{j}\right)\right|\right)=O\left(h^{\theta}\right) \quad(j=0,1, \ldots, N),
$$

where $\theta=\min (2,1+\alpha)$.

\subsection{The stability of fractional-order systems}

For a given fractional-order linear time-invariant system

$$
D_{*}^{\alpha} x=M x
$$

with $x(0)=x_{0}$, where $0<\alpha<1$ and $x \in R^{n}, M$ is a constant matrix.

Lemma 1 ([25]) System (11) is

(i) asymptotically stable if and only if

$$
\left|\arg \left(\lambda_{\ell}(M)\right)\right|>\frac{\alpha \pi}{2} \quad(\ell=1,2, \ldots, n),
$$

where $\arg \left(\lambda_{\ell}(M)\right)$ denotes the argument of the eigenvalue $\lambda_{\ell}$ of $M$. In this case, each component of the states decays toward 0 like $t^{-\alpha}$. 
(ii) stable if and only if

$$
\left|\arg \left(\lambda_{\ell}(M)\right)\right| \geq \frac{\alpha \pi}{2} \quad(\ell=1,2, \ldots, n)
$$

and those critical eigenvalues $\lambda_{i}$ that satisfy $\left|\arg \left(\lambda_{\ell}(M)\right)\right|=\alpha \pi / 2(\ell=1,2, \ldots, n)$, have geometric multiplicity one.

Fractional-order differential equations are at least as stable as their integer order counterpart because systems with memory are typically more stable than those without memory [23].

\section{CMGPS scheme of fractional-order chaotic real drive system and complex response system}

\subsection{Mathematical model and problem descriptions}

First, a class of $n$-dimensional fractional-order chaotic real drive systems is considered as

$$
D_{*}^{\alpha} x=M x+h(x)
$$

where $x=\left(x_{1}, x_{2}, \ldots, x_{n}\right)^{\mathrm{T}} \in \mathbb{R}^{n}$ is a real state vector, $M \in \mathbb{R}^{n \times n}$ is the coefficient matrix of $x$, while $h=\left(h_{1}, h_{2}, \ldots, h_{n}\right)^{\mathrm{T}}$ is a vector of complex nonlinear function.

The fractional-order complex chaotic response system with the controller is written as

$$
D_{*}^{\alpha} w=D_{*}^{\alpha} w^{r}+j D_{*}^{\alpha} w^{i}=B w+g(w)+v,
$$

where $w=w^{r}+j w^{i} \in \mathbb{C}^{n}$ and $w^{r}=\left(w_{1}^{r}, w_{2}^{r}, \ldots, w_{n}^{r}\right)^{\mathrm{T}} \in \mathbb{R}^{n}, w^{i}=\left(w_{1}^{i}, w_{2}^{i}, \ldots, w_{n}^{i}\right)^{\mathrm{T}} \in \mathbb{R}^{n}$, $B \in \mathbb{R}^{n \times n}$ is the coefficient matrix of $w$, while $g=\left(g_{1}, g_{2}, \ldots, g_{n}\right)^{\mathrm{T}}$ is a vector of complex nonlinear function, $v=v^{r}+j v^{i} \in \mathbb{C}^{n}$ is the controller to be designed.

Next the definition of CMGPS with complex transformation matrix is introduced for the fractional-order real chaotic drive system and the complex chaotic response system based on that of MGPS with real transformation matrix for two fractional-order real chaotic systems [13].

Definition 1 For the fractional-order real chaotic drive system (14) and the complex chaotic response system (15), it is said to be CMGPS with complex constant matrix $\Lambda=\Lambda^{r}+j \Lambda^{i}$ between $x(t)$ and $w(t)$ if there exists a controller $v=v^{r}+j v^{i} \in \mathbb{C}^{n}$ such that

$$
\lim _{t \rightarrow+\infty}\|w(t)-\Lambda x(t)\|=0
$$

i.e.,

$$
\lim _{t \rightarrow+\infty}\left\|w^{r}(t)-\Lambda^{r} x(t)\right\|=0
$$

and

$$
\lim _{t \rightarrow+\infty}\left\|w^{i}(t)-\Lambda^{i} x(t)\right\|=0
$$

while the matrix $\Lambda \in \mathbb{C}^{n \times n}$ is defined as a complex transformation matrix of the fractionalorder real chaotic drive system (14). 
If the error of CMGPS is defined as

$$
e(t)=e^{r}(t)+j e^{i}(t)=w(t)-\Lambda x(t)
$$

then

$$
\left\{\begin{array}{l}
e^{r}(t)=w^{r}(t)-\Lambda^{r} x(t), \\
e^{i}(t)=w^{i}(t)-\Lambda^{i} x(t),
\end{array}\right.
$$

the objective of this section is to design a controller $v$ to ensure that synchronization error tends to zero asymptotically, i.e.,

$$
\lim _{t \rightarrow+\infty}\left\|e^{r}(t)\right\|=0
$$

and

$$
\lim _{t \rightarrow+\infty}\left\|e^{i}(t)\right\|=0
$$

Remark 1 Most of the classical fractional-order real chaotic systems can be formed as system (14), such as fractional-order real Chua-Hartley's system [5], fractional-order real chaotic Lorenz-like system [6,11-13], and most of the classical fractional-order complex chaotic systems can be formed as system (15), such as fractional-order complex Lorenz system [14], fractional-order complex Chen system [15].

Remark 2 Several types of synchronization are special cases of CMGPS, such as complex modified projective synchronization (CMPS), complex projective synchronization (CPS), modified generalized projective synchronization (MGPS), modified projective synchronization (MPS), projective synchronization (PS), anti-synchronization (AS), complete synchronization (CS); see Table 1.

Therefore, the CMGPS will contain most existing works and extend previous works.

Remark 3 In particular, if the transformation matrix $\Lambda$ is zero, the CMGPS problem degenerates to the control problem of the fractional-order complex chaotic system (15).

Table 1 Types of synchronization

\begin{tabular}{ll}
\hline Settings of the matrix $\Lambda$ & Synchronization type \\
\hline$\Lambda=\Lambda^{r}+j \Lambda^{i} \in \mathbb{C}^{n \times n}$, non-diagonal & CMGPS \\
$\Lambda=\operatorname{diag}\left\{\delta_{1}, \delta_{2}, \ldots, \delta_{n}\right\} \in \mathbb{C}^{n \times n}$ & CMPS \\
$\Lambda=\operatorname{diag}\{\delta, \delta, \ldots, \delta\} \in \mathbb{C}^{n \times n}$ & CPS \\
$\Lambda \in \mathbb{R}^{n \times n}$, non-diagonal & MGPS \\
$\Lambda=\operatorname{diag}\left\{\delta_{1}, \delta_{2}, \ldots, \delta_{n}\right\} \in \mathbb{R}^{n \times n}$ & MPS \\
$\Lambda=\operatorname{diag}\{\delta, \delta, \ldots, \delta\} \in \mathbb{R}^{n \times n}$ & PS \\
$\Lambda=\operatorname{diag}\{-1,-1, \ldots,-1\}$ & AS \\
$\Lambda=\operatorname{diag}\{1,1, \ldots, 1\}$ & CS \\
\hline
\end{tabular}




\subsection{General method of CMGPS}

Theorem 1 For the given complex transformation matrix $\Lambda=\Lambda^{r}+j \Lambda^{i}$ and initial conditions $x(0), w(0)$, if the controller is designed as

$$
\begin{aligned}
v= & v^{r}+j v^{i}=(\Lambda M-B \Lambda) x-g(w)+\Lambda h(x)-K e \\
= & \left(\left(\Lambda^{r} M-B \Lambda^{r}\right) x-g^{r}(w)+\Lambda^{r} h(x)-K e^{r}\right) \\
& +j\left(\left(\Lambda^{i} M-B \Lambda^{i}\right) x-g^{i}(w)+\Lambda^{i}+h(x)-K e^{i}\right),
\end{aligned}
$$

then CMGPS between the fractional-order complex chaotic response system (15) and the real chaotic drive system (14) can be achieved with the desired complex transformation matrix $\Lambda$ asymptotically if and only if all the eigenvalues of $B-K$ satisfy $\left|\arg \left(\lambda_{\ell}(B-K)\right)\right|>$ $\frac{\alpha \pi}{2}(\ell=1,2, \ldots, n)$, where $K \in \mathbb{R}^{n \times n}$ is the control gain matrix.

Proof Equation (17) can be written as

$$
e(t)=e^{r}(t)+j e^{i}(t)=\left(w^{r}(t)-\Lambda^{r} x(t)\right)+j\left(w^{i}(t)-\Lambda^{i} x(t)\right) .
$$

Substituting Eq. (14) and Eq. (15) into Eq. (20), one can get the derivative of the error system

$$
\begin{aligned}
D_{*}^{\alpha} e= & D_{*}^{\alpha} e^{r}+j D_{*}^{\alpha} e^{i} \\
= & \left(D_{*}^{\alpha} w^{r}(t)-\Lambda^{r} D_{*}^{\alpha} x(t)\right)+j\left(D_{*}^{\alpha} w^{i}(t)-\Lambda^{i} D_{*}^{\alpha} x(t)\right) \\
= & \left(B w^{r}+g^{r}(w)-\Lambda^{r}(M x+h(x))+v^{r}\right) \\
& +j\left(B w^{i}+g^{i}(w)-\Lambda^{i}(M x+h(x))+v^{i}\right) .
\end{aligned}
$$

Insertion of (19) into Eq. (20) and separation of the real and imaginary parts give

$$
\left\{\begin{array}{l}
D_{*}^{\alpha} e^{r}=(B-K) e^{r}, \\
D_{*}^{\alpha} e^{i}=(B-K) e^{i} .
\end{array}\right.
$$

Due to Lemma 1, the error system (22) is asymptotically stable if and only if all the eigenvalues of $B-K$ satisfy $\left|\arg \left(\lambda_{\ell}(B-K)\right)\right|>\frac{\alpha \pi}{2}(\ell=1,2, \ldots, n)$, where $K \in \mathbb{R}^{n \times n}$ is the control gain matrix. That is, $\lim _{t \rightarrow+\infty}\left\|e^{r}(t)\right\|=0$, and $\lim _{t \rightarrow+\infty}\left\|e^{i}(t)\right\|=0$. Therefore, $\lim _{t \rightarrow+\infty}\|e(t)\|=0$, CMGPS between the fractional-order systems (14) and (15) is realized. This completes the proof.

\section{CMGPS scheme of fractional-order chaotic complex drive system and real response system}

\subsection{Mathematical model and problem descriptions}

Now, a class of $n$-dimensional fractional-order complex chaotic drive systems is considered as

$$
D_{*}^{\alpha} z=D_{*}^{\alpha} z^{r}+j D_{*}^{\alpha} z^{i}=A z+f(z)
$$

where $z=z^{r}+j z^{i} \in \mathbb{C}^{n}$ and $z^{r}=\left(z_{1}^{r}, z_{2}^{r}, \ldots, z_{n}^{r}\right)^{\mathrm{T}} \in \mathbb{R}^{n}, z^{i}=\left(z_{1}^{i}, z_{2}^{i}, \ldots, z_{n}^{i}\right)^{\mathrm{T}} \in \mathbb{R}^{n}, A \in \mathbb{R}^{n \times n}$ are the coefficient matrix of $z$, while $f=\left(f_{1}, f_{2}, \ldots, f_{n}\right)^{\mathrm{T}}$ is a vector of complex nonlinear function. 
The fractional-order real chaotic response system with the controller is written as

$$
D_{*}^{\alpha} y=H y+p(y)+v,
$$

where $y=\left(y_{1}, y_{2}, \ldots, y_{n}\right)^{\mathrm{T}} \in \mathbb{R}^{n}$ is a real state vector, $H \in \mathbb{R}^{n \times n}$ is the coefficient matrix of $y$, while $p=\left(p_{1}, p_{2}, \ldots, p_{n}\right)^{\mathrm{T}}$ is a vector of complex nonlinear function, $v=\left(v_{1}, v_{2}, \ldots, v_{n}\right)^{\mathrm{T}} \in$ $\mathbb{R}^{n}$ is the controller to be designed.

Next the definition of CMGPS with complex transformation matrix is introduced for the fractional-order complex chaotic drive system and the real chaotic response system based on that of MGPS with real transformation matrix for two fractional-order real chaotic systems [13].

Definition 2 For the fractional-order complex chaotic drive system (23) and the real chaotic response system (24), it is said to be CMGPS with constant matrix $\Lambda=\Lambda^{r}+j \Lambda^{i}$ between $z(t)$ and $y(t)$ if there exists a controller $v$ such that

$$
\lim _{t \rightarrow+\infty}\left\|y(t)-\Lambda^{r} z^{r}(t)+\Lambda^{i} z^{i}(t)\right\|=0
$$

while the matrix $\Lambda \in \mathbb{C}^{n \times n}$ is defined as a complex transformation matrix of the fractionalorder complex chaotic drive system (23).

If the error of CMGPS is defined as

$$
e(t)=y(t)-\Lambda^{r} z^{r}(t)+\Lambda^{i} z^{i}(t)
$$

the objective of this section is to design a controller $v$ to ensure that synchronization error (26) tends to zero asymptotically, i.e.,

$$
\lim _{t \rightarrow+\infty}\|e(t)\|=0
$$

\subsection{General method of CMGPS}

Theorem 2 For the given complex transformation matrix $\Lambda=\Lambda^{r}+j \Lambda^{i}$ and initial conditions $z(0), y(0)$, if the designed controller is

$$
v=\left(\Lambda^{r} A-H \Lambda^{r}\right) z^{r}-\left(\Lambda^{i} A-H \Lambda^{i}\right) z^{i}-p(y)+\Lambda^{r} f^{r}(z)-\Lambda^{i} f^{i}(z)-K e,
$$

then CMGPS between the fractional-order real chaotic response system (24) and the complex drive system (23) can be achieved with the desired complex transformation matrix $\Lambda$ asymptotically if and only if all the eigenvalues of $H-K$ satisfy $\left|\arg \left(\lambda_{\ell}(H-K)\right)\right|>\frac{\alpha \pi}{2}$ $(\ell=1,2, \ldots, n)$, where $K \in \mathbb{R}^{n \times n}$ is the control gain matrix.

Proof Substituting Eq. (23) and Eq. (24) into Eq. (26), one can get the derivative of the error system

$$
\begin{aligned}
D_{*}^{\alpha} e & =D_{*}^{\alpha} y-\Lambda^{r} D_{*}^{\alpha} z^{r}+\Lambda^{i} D_{*}^{\alpha} z^{i}=H y+p(y)+v-\Lambda^{r}\left(A z^{r}+f^{r}(z)\right)+\Lambda^{i}\left(A z^{i}+f^{i}(z)\right) \\
& =H e+H\left(\Lambda^{r} z^{r}-\Lambda^{i} z^{i}\right)+p(y)+v-\Lambda^{r}\left(A z^{r}+f^{r}(z)\right)+\Lambda^{i}\left(A z^{i}+f^{i}(z)\right) .
\end{aligned}
$$


Insertion of Eq. (27) into Eq. (28) gives

$$
D_{*}^{\alpha} e=(H-K) e .
$$

Due to Lemma 1, the error system (29) is asymptotically stable if and only if all the eigenvalues of $H-K$ satisfy $\left|\arg \left(\lambda_{\ell}(H-K)\right)\right|>\frac{\alpha \pi}{2}(\ell=1,2, \ldots, n)$, where $K \in \mathbb{R}^{n \times n}$ is the control gain matrix. That is, $\lim _{t \rightarrow+\infty}\|e(t)\|=0$, the fractional-order real chaotic response system (24) and the complex chaotic drive system (23) realize CMGPS. This completes the proof.

\section{CMGPS scheme of two fractional-order chaotic complex systems}

\subsection{Mathematical model and problem descriptions}

Now, the definition of CMGPS with complex transformation matrix is introduced for two fractional-order complex chaotic systems based on that of MGPS with real transformation matrix for two fractional-order real chaotic systems [13].

Definition 3 For the fractional-order complex chaotic drive system (23) and the response system (15), it is said to be CMGPS with complex matrix $\Lambda=\Lambda^{r}+j \Lambda^{i}$ between $z(t)$ and $w(t)$ if there exists a controller $v=v^{r}+j v^{i} \in \mathbb{C}^{n}$ such that

$$
\lim _{t \rightarrow+\infty}\|w(t)-\Lambda z(t)\|=0
$$

i.e.,

$$
\lim _{t \rightarrow+\infty}\left\|w^{r}(t)-\Lambda^{r} z^{r}(t)+\Lambda^{i} z^{i}(t)\right\|=0,
$$

and

$$
\lim _{t \rightarrow+\infty}\left\|w^{i}(t)-\Lambda^{r} z^{i}(t)-\Lambda^{i} z^{r}(t)\right\|=0
$$

while the matrix $\Lambda \in \mathbb{C}^{n \times n}$ is defined as a complex transformation matrix of the fractionalorder complex chaotic drive system (23).

If the error of CMGPS is defined as

$$
e(t)=e^{r}(t)+j e^{i}(t)=w(t)-\Lambda z(t)
$$

then

$$
\left\{\begin{array}{l}
e^{r}(t)=w^{r}(t)-\Lambda^{r} z^{r}(t)+\Lambda^{i} z^{i}(t), \\
e^{i}(t)=w^{i}(t)-\Lambda^{r} z^{i}(t)-\Lambda^{i} z^{r}(t),
\end{array}\right.
$$

the objective of this section is to design a controller $v$ to ensure that synchronization error tends to zero asymptotically, i.e.,

$$
\lim _{t \rightarrow+\infty}\left\|e^{r}(t)\right\|=0
$$


and

$$
\lim _{t \rightarrow+\infty}\left\|e^{i}(t)\right\|=0
$$

\subsection{General method of CMGPS}

Theorem 3 For the given complex transformation matrix $\Lambda=\Lambda^{r}+j \Lambda^{i}$ and initial conditions $z(0), w(0)$, if the controller is designed as

$$
\begin{aligned}
\nu= & v^{r}+j v^{i} \\
= & (\Lambda A-B \Lambda) z-g(w)+\Lambda f(z)-K e \\
= & \left(\Lambda^{r} A-B \Lambda^{r}\right) z^{r}-\left(\Lambda^{i} A-B \Lambda^{i}\right) z^{i}-g^{r}(w)+\Lambda^{r} f^{r}(z)-\Lambda^{i} f^{i}(z)-K e^{r} \\
& +j\left(\left(\Lambda^{i} A-B \Lambda^{i}\right) z^{r}+\left(\Lambda^{r} A-B \Lambda^{r}\right) z^{i}-g^{i}(w)+\Lambda^{i} f^{r}(z)+\Lambda^{r} f^{i}(z)-K e^{i}\right),
\end{aligned}
$$

then CMGPS between the fractional-order complex chaotic response system (15) and the drive system (23) can be achieved with the desired complex transformation matrix $\Lambda$ asymptotically if and only if all the eigenvalues of $B-K$ satisfy $\left|\arg \left(\lambda_{\ell}(B-K)\right)\right|>\frac{\alpha \pi}{2}$ $(\ell=1,2, \ldots, n)$, where $K \in \mathbb{R}^{n \times n}$ is the control gain matrix.

Proof It is similar to the proof in Theorem 1 and thus is omitted.

Corollary 1 If the structure of systems (15) and (23) is identical, i.e., $A=B$, and $f=g$, and the controller is designed as

$$
\begin{aligned}
v= & v^{r}+j v^{i}=(\Lambda A-A \Lambda) z-f(w)+\Lambda f(z)-K e \\
= & \left(\Lambda^{r} A-A \Lambda^{r}\right) z^{r}-\left(\Lambda^{i} A-A \Lambda^{i}\right) z^{i}-f^{r}(w)+\Lambda^{r} f^{r}(z)-\Lambda^{i} f^{i}(z)-K e^{r} \\
& +j\left(\left(\Lambda^{i} A-A \Lambda^{i}\right) z^{r}+\left(\Lambda^{r} A-A \Lambda^{r}\right) z^{i}-f^{i}(w)+\Lambda^{i} f^{r}(z)+\Lambda^{r} f^{i}(z)-K e^{i}\right),
\end{aligned}
$$

then CMGPS between the fractional-order complex chaotic response system (15) and the drive system (23) can be achieved with the desired complex transformation matrix $\Lambda$ asymptotically if and only if all the eigenvalues of $B-K$ satisfy $\left|\arg \left(\lambda_{\ell}(B-K)\right)\right|>\frac{\alpha \pi}{2}$ $(\ell=1,2, \ldots, n)$, where $K \in \mathbb{R}^{n \times n}$ is the control gain matrix.

Remark 4 In particular, if the transformation matrix $\Lambda=\operatorname{diag}\{1,1, \ldots, 1\}$, then CMGPS in Corollary 1 is reduced to complete synchronization (CS) of identical fractional-order complex chaotic systems in [14].

\section{Numerical examples}

Now, three examples are worked out to illustrate the theoretical results in this paper.

\subsection{CMGPS of fractional-order chaotic real Chua-Hartley's drive system and complex Lorenz response system}

In order to illustrate CMGPS behaviors of a fractional-order real chaotic drive system and a complex chaotic response system, it is assumed that the fractional-order chaotic 


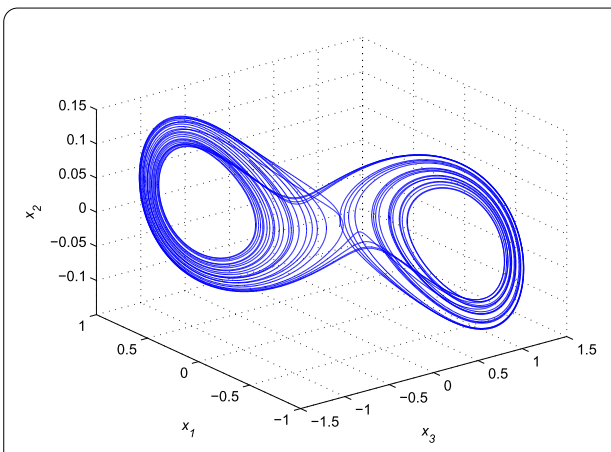

(a) The chaotic attractor of system (35) when $\alpha=0.94$

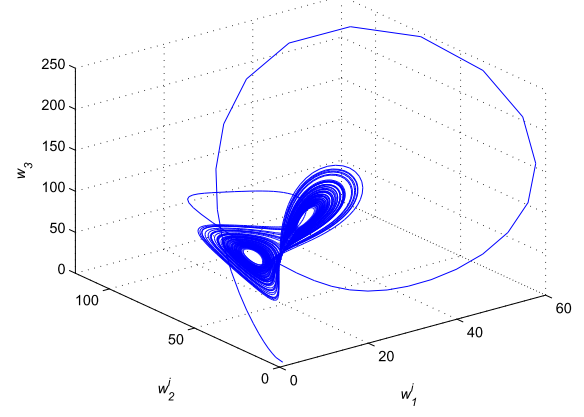

(c) The chaotic attractor of system (36) when $\alpha=0.96$

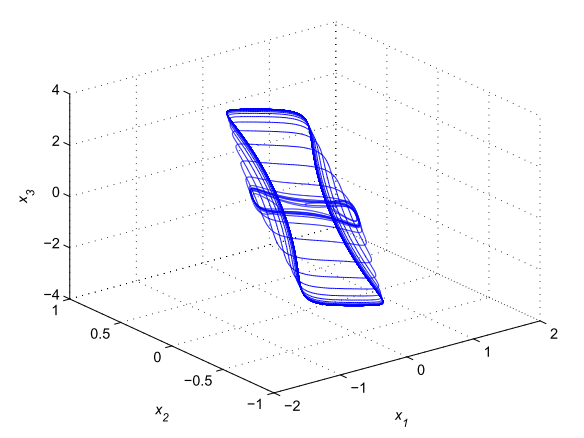

(b) The chaotic attractor of system (35) when $\alpha=0.96$

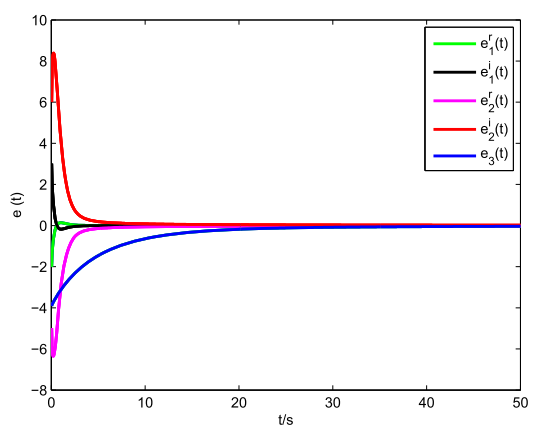

(d) The dynamics of CMGPS error when $\alpha=0.96$

Figure 1 The CMGPS of fractional-order real chaotic Chua-Hartley's drive system (35) and complex chaotic Lorenz response system (36).

real Chua-Hartley's system [5] drives the fractional-order chaotic complex Lorenz system [14]. Therefore, the drive system is given in the form

$$
\left\{\begin{array}{l}
D_{*}^{\alpha} x_{1}=m_{1}\left(x_{2}+\frac{x_{1}-2 x_{1}^{3}}{7}\right) \\
D_{*}^{\alpha} x_{2}=x_{1}-x_{2}+x_{3} \\
D_{*}^{\alpha} x_{3}=-m_{2} x_{2}
\end{array}\right.
$$

where $x=\left(x_{1}, x_{2}, x_{3}\right)^{T} \in \mathbb{R}^{3}$ is a real state vector. When $m_{1}=12.75, m_{2}=\frac{100}{7}$, the fractional order Chua-Hartley's system (35) is chaotic as in Figure 1(a) and Figure 1(b) at $\alpha=0.94$ and $\alpha=0.96$, respectively.

The response system with the controller is written in the form

$$
\left\{\begin{array}{l}
D_{*}^{\alpha} w_{1}=b_{1}\left(w_{2}-w_{1}\right)+v_{1} \\
D_{*}^{\alpha} w_{2}=b_{2} w_{1}-w_{2}-w_{1} w_{3}+v_{2}, \\
D_{*}^{\alpha} w_{3}=-b_{3} w_{3}+(1 / 2)\left(\bar{w}_{1} w_{2}+w_{1} \bar{w}_{2}\right)+v_{3},
\end{array}\right.
$$

where $w_{1}=w_{1}^{r}+j w_{1}^{i}, w_{2}=w_{2}^{r}+j w_{2}^{i}$ are complex state variables, and $w_{3}$ is a real state variable. System (36) is chaotic when $b_{1}=10, b_{2}=160, b_{3}=1, \alpha=0.96$ and in the absence of the controller $v=v^{r}+j v^{i}$ as in Figure 1(c), see [14] for more details. 
The complex transformation matrix can be taken as

$$
\Lambda=\left(\begin{array}{ccc}
1-j & 0 & 0 \\
0 & 1+j & 0 \\
0 & 1 & -1
\end{array}\right)=\left(\begin{array}{ccc}
1 & 0 & 0 \\
0 & 1 & 0 \\
0 & 1 & -1
\end{array}\right)+j\left(\begin{array}{ccc}
-1 & 0 & 0 \\
0 & 1 & 0 \\
0 & 0 & 0
\end{array}\right)
$$

and the error system $e(t)=w(t)-\Lambda x(t)$ is obtained as

$$
\left\{\begin{array}{l}
e_{1}=w_{1}-(1-j) x_{1}=\left(w_{1}^{r}-x_{1}\right)+j\left(w_{1}^{i}+x_{1}\right) \\
e_{2}=w_{2}-(1+j) x_{2}=\left(w_{2}^{r}-x_{2}\right)+j\left(w_{2}^{i}-x_{2}\right), \\
e_{3}=w_{3}-x_{2}+x_{3}
\end{array}\right.
$$

The control gain matrix is chosen as

$$
K=\left(\begin{array}{ccc}
6 & 11 & 0 \\
120 & 3 & 0 \\
0 & 0 & 0
\end{array}\right)
$$

and the controller is designed according to (19) in Theorem 1 as follows:

$$
\begin{aligned}
v= & \left(\begin{array}{c}
\left(b_{1}+\frac{m_{1}}{7}\right) x_{1}-\left(b_{1}-m_{1}\right) x_{2}-\frac{2}{7} m_{1} x_{1}{ }^{3}-6 e_{1}^{r}-11 e_{2}^{r} \\
\left(1-b_{2}\right) x_{1}+x_{3}+w_{1}^{r} w_{3}-120 e_{1}^{r}-3 e_{2}^{r} \\
x_{1}+\left(b_{3}-m_{2}-1\right) x_{2}+\left(1-b_{3}\right) x_{3}-\left(w_{1}^{r} w_{2}^{r}+w_{1}^{i} w_{2}^{i}\right)
\end{array}\right) \\
& +j\left(\begin{array}{c}
-\left(b_{1}+\frac{m_{1}}{7}\right) x_{1}-\left(b_{1}+m_{1}\right) x_{2}+\frac{2}{7} m_{1} x_{1}^{3}-6 e_{1}^{i}-11 e_{2}^{i} \\
\left(1+b_{2}\right) x_{1}+x_{3}+w_{1}^{i} w_{3}-120 e_{1}^{i}-3 e_{2}^{i} \\
0
\end{array}\right) .
\end{aligned}
$$

The parameters of drive system (35) and response system (36) are selected as $\alpha=0.96$, $m_{1}=12.75, m_{2}=\frac{100}{7}$, and $b_{1}=10, b_{2}=160, b_{3}=1$, respectively. The initial values are randomly chosen as $x_{0}=(0.01,-0.01,0.1)^{T}$ and $w_{0}=w_{0}^{r}+j w_{0}^{i}=(-2+3 j,-5+6 j,-4)^{T}$, respectively. Therefore, all of the eigenvalues of $B-K$ are $\lambda_{1}=-10+2 j, \lambda_{2}=-10-2 j$, $\lambda_{3}=-1$, which satisfies $\left|\arg \left(\lambda_{\ell}(B-K)\right)\right|>\frac{\alpha \pi}{2}(\ell=1,2,3)$. The errors of CMGPS converge asymptotically to zero as in Figure 1(d). Hence, CMGPS has been achieved between the fractional-order real chaotic Chua-Hartley's drive system (35) and the complex chaotic Lorenz response system (36).

\subsection{CMGPS of fractional-order chaotic complex Chen drive system and real Chua-Hartley's response system}

In order to illustrate CMGPS behaviors of a fractional-order complex chaotic drive system and a real chaotic response system, it is assumed that the fractional-order complex chaotic Chen system [15] drives the fractional-order real chaotic Chua-Hartley's system [6]. Therefore, the drive system is written in the form

$$
\left\{\begin{array}{l}
D_{*}^{\alpha} z_{1}=a_{1}\left(z_{2}-z_{1}\right) \\
D_{*}^{\alpha} z_{2}=\left(a_{3}-a_{1}\right) z_{1}+a_{3} z_{2}-z_{1} z_{3} \\
D_{*}^{\alpha} z_{3}=-a_{2} z_{3}+(1 / 2)\left(\bar{z}_{1} z_{2}+z_{1} \bar{z}_{2}\right)
\end{array}\right.
$$




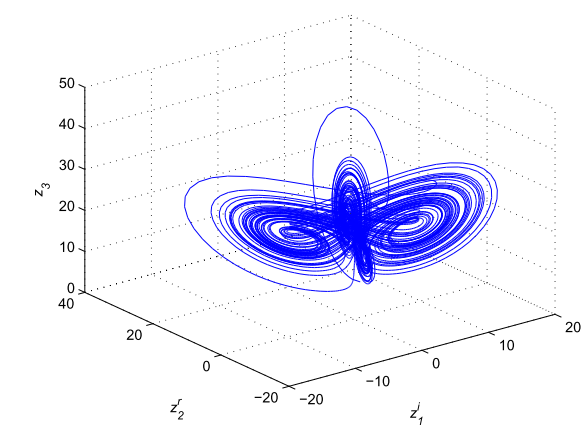

(a) The chaotic attractor of system (39)

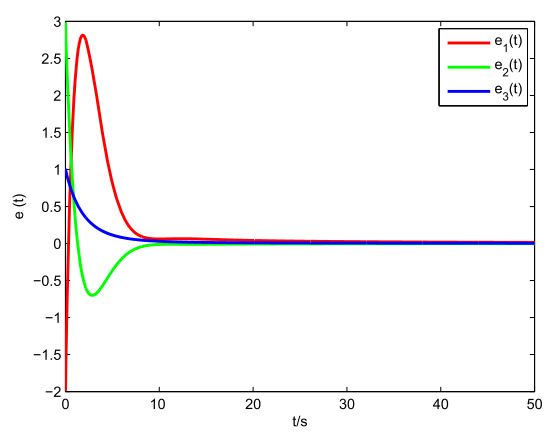

(b) The dynamics of CMGPS error

Figure 2 The CMGPS of fractional-order complex chaotic Chen drive system (39) and real chaotic Chua-Hartley's response system (40) when $\alpha=0.96$.

where $z_{1}=z_{1}^{r}+j z_{1}^{i}, z_{2}=z_{2}^{r}+j z_{2}^{i}$ are complex state variables and $z_{3}$ is a real state variable. System (39) is chaotic when $a_{1}=35, a_{2}=3, a_{3}=28, \alpha=0.96$ in Figure 2(a), see [15] for more details.

The response system with the controller is given in the form

$$
\left\{\begin{array}{l}
D_{*}^{\alpha} y_{1}=h_{1}\left(y_{2}+\frac{y_{1}-2 y_{1}^{3}}{7}\right)+v_{1} \\
D_{*}^{\alpha} y_{2}=y_{1}-y_{2}+y_{3}+v_{2} \\
D_{*}^{\alpha} y_{3}=-h_{2} y_{2}+v_{3}
\end{array}\right.
$$

where $y=\left(y_{1}, y_{2}, y_{3}\right)^{T} \in \mathbb{R}^{3}$ is a real state vector, $v=\left(v_{1}, v_{2}, v_{3}\right)^{T} \in \mathbb{R}^{3}$ is the controller.

The complex transformation matrix can be taken as

$$
\Lambda=\left(\begin{array}{ccc}
1 & 0 & 0 \\
0 & -j & 0 \\
j & 0 & -1
\end{array}\right)=\left(\begin{array}{ccc}
1 & 0 & 0 \\
0 & 0 & 0 \\
0 & 0 & -1
\end{array}\right)+j\left(\begin{array}{ccc}
0 & 0 & 0 \\
0 & -1 & 0 \\
1 & 0 & 0
\end{array}\right) \text {, }
$$

and the error system $e(t)=y(t)-\Lambda^{r} z^{r}(t)+\Lambda^{i} z^{i}(t)$ is obtained as

$$
\left\{\begin{array}{l}
e_{1}=y_{1}-z_{1}^{r} \\
e_{2}=y_{2}+z_{2}^{i} \\
e_{3}=y_{3}-z_{1}^{i}+z_{3}
\end{array}\right.
$$

The control gain matrix is chosen as

$$
K=\left(\begin{array}{ccc}
\frac{5.75}{7} & 7.75 & 0 \\
2 & 2 & 0 \\
0 & -\frac{100}{7} & 1
\end{array}\right)
$$

and the real controller is designed according to (27) in Theorem 2 as follows:

$$
\left\{\begin{array}{l}
v_{1}=-\left(\frac{1}{7} h_{1}+a_{1}\right) z_{1}^{r}+a_{1} z_{2}^{r}+h_{1} z_{2}^{i}+\frac{2}{7} h_{1} y_{1}^{3}-\frac{5.75}{7} e_{1}-7.75 e_{2}, \\
v_{2}=-z_{1}^{r}-\left(a_{3}-a_{1}+1\right) z_{1}^{i}-\left(a_{3}+1\right) z_{2}^{i}+z_{1}^{i} z_{3}+z_{3}-2 e_{1}-2 e_{2}, \\
v_{3}=-a_{1} z_{1}^{i}+\left(a_{1}-h_{2}\right) z_{2}^{i}+a_{2} z_{3}-\left(z_{1}^{r} z_{2}^{r}+z_{1}^{i} z_{2}^{i}\right)+\frac{100}{7} e_{2}-e_{3} .
\end{array}\right.
$$


The parameters of drive system (39) and response system (40) are selected as $\alpha=0.96$, $a_{1}=35, a_{2}=3, a_{3}=28$, and $h_{1}=12.75, h_{2}=\frac{100}{7}$, respectively. The initial values are randomly chosen as $z_{0}=z_{0}^{r}+j z_{0}^{i}=(2+4 j, 6+3 j, 5)^{T}$ and $y_{0}=(0.01,-0.01,0.1)^{T}$, respectively. Therefore, all of the eigenvalues of $H-K$ are $\lambda_{1}=-1+j, \lambda_{2}=-1-j, \lambda_{3}=-1$, which satisfies $\left|\arg \left(\lambda_{\ell}(H-K)\right)\right|>\frac{\alpha \pi}{2}(\ell=1,2,3)$. The errors of CMGPS converge asymptotically to zero as in Figure 2(b). Hence, CMGPS has been achieved between the fractional-order complex chaotic Chen drive system (39) and the real chaotic Chua-Hartley's response system (40).

\subsection{CMGPS of fractional-order chaotic complex Chen drive system and complex Lorenz response system}

In order to illustrate CMGPS behaviors of two fractional-order chaotic complex systems, it is assumed that the fractional-order complex chaotic Chen system (39) drives the fractional-order complex chaotic Lorenz system (36). The parameters of drive system (39) and response system (36) are selected as $\alpha=0.96, a_{1}=35, a_{2}=3, a_{3}=28$, and $b_{1}=10$, $b_{2}=160, b_{3}=1$, respectively.

The complex transformation matrix can be taken as

$$
\Lambda=\left(\begin{array}{ccc}
-j & 0 & 0 \\
-1 & 2 j & 0 \\
0 & 0 & -2
\end{array}\right)=\left(\begin{array}{ccc}
0 & 0 & 0 \\
-1 & 0 & 0 \\
0 & 0 & -2
\end{array}\right)+j\left(\begin{array}{ccc}
-1 & 0 & 0 \\
0 & 2 & 0 \\
0 & 0 & 0
\end{array}\right)
$$

and the error system $e(t)=w(t)-\Lambda z(t)$ is obtained as

$$
\left\{\begin{array}{l}
e_{1}=w_{1}-(-j) z_{1}=\left(w_{1}^{r}-z_{1}^{i}\right)+j\left(w_{1}^{i}+z_{1}^{r}\right), \\
e_{2}=w_{2}+z_{1}-2 j z_{2}=\left(w_{2}^{r}+z_{1}^{r}+2 z_{2}^{i}\right)+j\left(w_{2}^{i}+z_{1}^{i}-2 z_{2}^{r}\right), \\
e_{3}=w_{3}+2 z_{3} .
\end{array}\right.
$$

The control gain matrix is chosen as

$$
K=\left(\begin{array}{ccc}
-1 & 10.25 & 0 \\
60 & 0 & 0 \\
0 & 0 & 0
\end{array}\right)
$$

and the complex controller is designed according to (33) in Theorem 3 as follows:

$$
\begin{aligned}
v= & \left(\begin{array}{c}
10 z_{1}^{r}-25 z_{1}^{i}+55 z_{2}^{i}+e_{1}^{r}-10.25 e_{2}^{r} \\
34 z_{1}^{r}-146 z_{1}^{i}-35 z_{2}^{r}-58 z_{2}^{i}+2 z_{1}^{i} z_{3}+w_{1}^{r} w_{3}-60 e_{1}^{r} \\
4 z_{3}-2\left(z_{1}^{r} z_{2}^{r}+z_{1}^{i} z_{2}^{i}\right)-\left(w_{1}^{r} w_{2}^{r}+w_{1}^{i} w_{2}^{i}\right)
\end{array}\right) \\
& +j\left(\begin{array}{c}
25 z_{1}^{r}+10 z_{1}^{i}-55 z_{2}^{r}+e_{1}^{i}-10.25 e_{2}^{i} \\
\left.146 z_{1}^{r}+34 z_{1}^{i}+58 z_{2}^{r}-35 z_{2}^{i}-2 z_{1}^{r} z_{3}+w_{1}^{i} w_{3}-60 e_{1}^{i}\right) \\
0
\end{array}\right) .
\end{aligned}
$$

The initial values are randomly chosen as $z_{0}=(2+4 j, 6+3 j, 5)^{T}$ and $w_{0}=w_{0}^{r}+j w_{0}^{i}=(-2+$ $3 j,-5+6 j,-4)^{T}$, respectively. Therefore, all of the eigenvalues of $B-K$ are $\lambda_{1}=-5+3 j, \lambda_{2}=$ $-5-3 j, \lambda_{3}=-1$, which satisfies $\left|\arg \left(\lambda_{\ell}(B-K)\right)\right|>\frac{\alpha \pi}{2}(\ell=1,2,3)$. The errors of CMGPS converge asymptotically to zero as in Figure 3 , where the blue line shows the real parts of 
Figure 3 The CMGPS error dynamics of fractional-order chaotic complex Chen drive system (39) and complex Lorenz response system (36) with the controller (44) when $\alpha=0.96$.

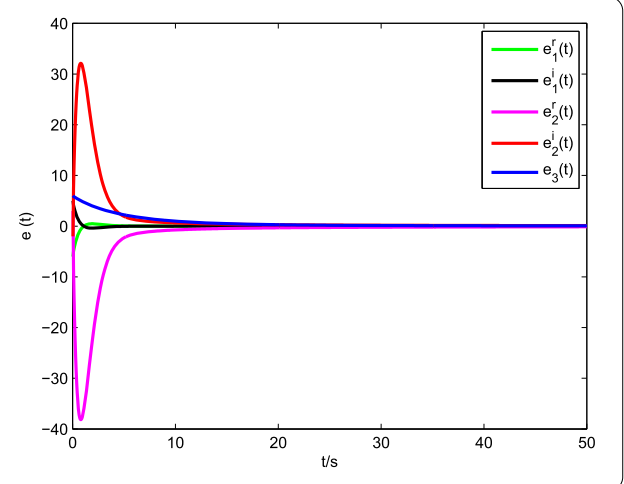

the errors and the red line presents the imaginary parts of the errors. Hence, CMGPS has been achieved between the fractional-order chaotic complex Chen drive system (39) and the complex Lorenz response system (36).

\section{Conclusions}

In this paper, the definitions of modified generalized projective synchronization with complex transformation matrix (CMGPS) are introduced, where the drive and response systems could be asymptotically synchronized up to a desired complex transformation matrix, not a diagonal matrix. Moreover, general methods of CMGPS are designed for the fractional-order real chaotic drive system and the complex chaotic response system, and for the fractional-order complex chaotic drive system and the real chaotic response system, and for two fractional-order complex chaotic systems, respectively. It should be noticed that the Lyapunov function is not required to be calculated in this scheme; it is really simple and feasible in practical applications. Three numerical examples are worked out to illustrate the feasibility and effectiveness of the theoretical results.

The complex transformation matrix not only establishes a link between fractional-order real chaotic systems and complex chaotic systems, but also sets up a bridge between two fractional-order complex chaotic systems. It increases the range of choosing fractionalorder chaotic generators in the transmitters and receivers, thus an interceptor is harder to crack information sources.

Competing interests

The authors declare that they have no competing interests.

Authors' contributions

The authors declare that the study was realized in collaboration with the same responsibility. All authors read and approved the final manuscript.

\section{Author details}

${ }^{1}$ School of Mathematical Sciences, University of Ji'nan, Ji'nan, Shandong 250022, P.R. China. ${ }^{2}$ School of Control Science and Engineering, Shandong University, Ji'nan, Shandong 250061, P.R. China. ${ }^{3}$ Public Basic Department, Shandong Radio and TV University, Ji'nan, Shandong 250010, P.R. China.

\section{Acknowledgements}

This research was partially supported by the National Nature Science Foundation of China (Grant Nos. 61273088, 61473133), the Nature Science Foundation of Shandong Province, China (No. ZR2014FL015), and the Foundation for University Young Key Teacher Program of Shandong Provincial Education Department, China.

Received: 20 January 2015 Accepted: 27 July 2015 Published online: 04 September 2015

\section{References}

1. Bagley, RL, Calico, RA: Fractional-order state equations for the control of viscoelastically damped structures. J. Guid. Control Dyn. 14(2), 304-311 (1991) 
2. Sun, $\mathrm{HH}$, Abdelwahad, AA, Onaral, B: Linear approximation of transfer function with a pole of fractional-order. IEEE Trans. Autom. Control 29(5), 441-444 (1984)

3. Laskin, N: Fractional market dynamics. Physica A 287(3-4), 482-492 (2000)

4. Kunsezov, D, Bulagc, A, Dang, GD: Quantum Levy processes and fractional kinetics. Phys. Rev. Lett. 82(6), 1136-1139 (1999)

5. Hartley, TT, Lorenzo, CF, Qammer, HK: Chaos in a fractional-order Chua's system. IEEE Trans. Circuits Syst. I 42(8), 485-490 (1995)

6. Arena, P, Caponetto, R, Fortuna, L, Porto, D: Bifurcation and chaos in noninteger order cellular neural networks. Int. J. Bifurc. Chaos 8(7), 1527-1539 (1998)

7. Gao, X, Yu, J: Chaos in the fractional order periodically forced complex Duffing's oscillators. Chaos Solitons Fractals 24 1097-1104 (2005)

8. Wu, GC, Baleanu, D: Discrete fractional logistic map and its chaos. Nonlinear Dyn. 75(1-2), 283-287 (2014)

9. Wu, GC, Baleanu, D: Chaos synchronization of the discrete fractional logistic map. Signal Process. 102, 96-99 (2014)

10. Wu, GC, Baleanu, D, Zeng, SD: Discrete chaos in fractional sine and standard maps. Phys. Lett. A 378(5-6), 484-487 (2014)

11. Wang, $X Y, H e, Y$ J: Projective synchronization of fractional order chaotic system based on linear separation. Phys. Lett. A 372(4), 435-441 (2008)

12. $\mathrm{Wu}, \mathrm{XJ}, \mathrm{Lu}, \mathrm{Y}:$ Generalized projective synchronization of the fractional-order Chen hyperchaotic system. Nonlinear Dyn. 57(1-2), 25-35 (2009)

13. Liu, J, Liu, ST, Yuan, CH: Modified generalized projective synchronization of fractional-order chaotic Lü systems. Adv. Differ. Equ. 2013, Article ID 374 (2013)

14. Luo, C, Wang, XY: Chaos in the fractional-order complex Lorenz system and its synchronization. Nonlinear Dyn. 71, 241-257 (2013)

15. Luo, C, Wang, XY: Chaos generated from the fractional-order complex Chen system and its application to digital secure communication. Int. J. Mod. Phys. C 24(4), 1350025 (2013)

16. Mahmoud, GM, Mahmoud, EE: Complex modified projective synchronization of two chaotic complex nonlinear systems. Nonlinear Dyn. 73(4), 2231-2240 (2013)

17. Zhang, FF, Liu, ST, Yu, WY: Modified projective synchronization with complex scaling factors of uncertain real chaos and complex chaos. Chin. Phys. B 22, Article ID 120505 (2013)

18. Liu, J, Liu, ST, Zhang, FF: A novel four-wing hyperchaotic complex system and its complex modified hybrid projective synchronization with different dimensions. Abstr. Appl. Anal. 2014, Article ID 257327 (2014)

19. Liu, J, Liu, ST, Yuan, CH: Adaptive complex modified projective synchronization of complex chaotic (hyperchaotic) systems with uncertain complex parameters. Nonlinear Dyn. 79, 1035-1047 (2015)

20. Liu, J: Complex modified hybrid projective synchronization of different dimensional fractional-order complex chaos and real hyper-chaos. Entropy 16, 6195-6211 (2014)

21. Podlubny, l: Fractional Differential Equations. Academic Press, New York (1999)

22. Deng, WH, Li, CP: Chaos synchronization of the fractional Lü system. Physica A 353, 61-72 (2005)

23. Diethelm, K, Ford, NJ, Freed, AD: A predictor-corrector approach for the numerical solution of fractional differential equations. Nonlinear Dyn. 29(1-4), 3-22 (2002)

24. Li, C, Peng, GJ: Chaos in Chen's system with a fractional order. Chaos Solitons Fractals 22(2), 443-450 (2004)

25. Matignon, D: Stability results for fractional differential equations with applications to control processing. In: Proceedings of the IMACS-SMC. Lille, France (1996)

\section{Submit your manuscript to a SpringerOpen ${ }^{\odot}$ journal and benefit from:}

$\checkmark$ Convenient online submission

- Rigorous peer review

- Immediate publication on acceptance

Open access: articles freely available online

- High visibility within the field

- Retaining the copyright to your article 